Actes des congrès de la Société française

5 | 1984

Mythe et histoire

\title{
Mythes de la Renaissance
}

\section{Yves Peyré}

\section{(Q) OpenEdition \\ Journals}

Édition électronique

URL : http://journals.openedition.org/shakespeare/494

DOI : 10.4000/shakespeare.494

ISSN : 2271-6424

Éditeur

Société Française Shakespeare

Édition imprimée

Date de publication : 1 novembre 1984

Pagination : 67-94

Référence électronique

Yves Peyré, "Mythes de la Renaissance », Actes des congrès de la Société française Shakespeare [En ligne], 5 | 1984, mis en ligne le 01 janvier 2007, consulté le 05 mai 2019. URL : http:// journals.openedition.org/shakespeare/494; DOI : 10.4000/shakespeare.494 
SOCIETE FRANÇAISE SHAKESPEARE

Actes du Congrès 1983

\section{MYTHE ET HISTOIRE}

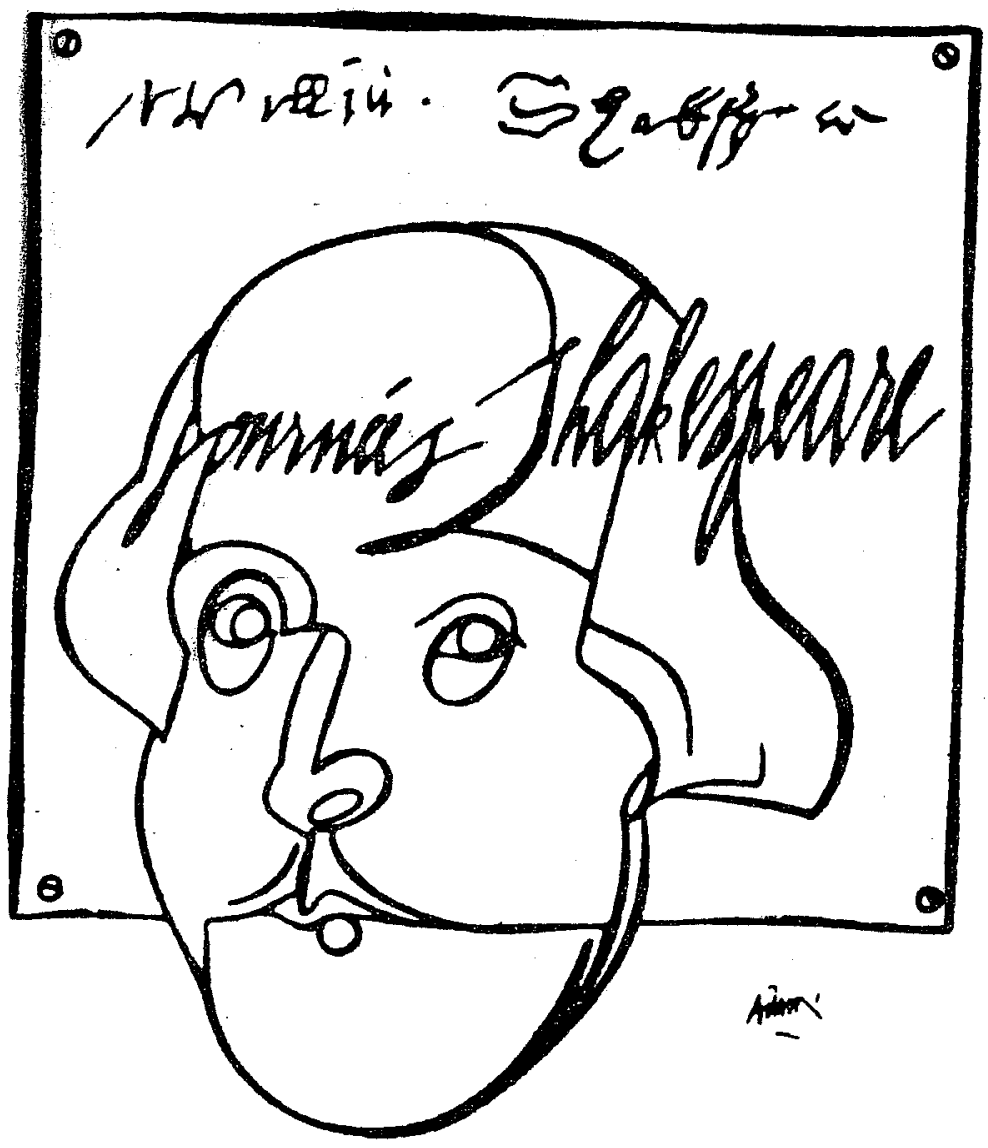

$$
\begin{array}{r}
\text { DiReCteur DE LA' PUblicatic } \\
\text { M.T. Jones-Davie }
\end{array}
$$

Publié avec le concours du Centre National de la Recherche Scientifiq

$$
\text { JEAN TOUZOT Libraire - Editeur }
$$

38, rue Saint-Sulpice 75278 PARIS CEDEX 06199 


\section{MYTHES DE RENAISSANCE}

And every thing in May revarts.

Alexander Scott

Only death adds to'our length : nor are we grown In stature to be men, till we are none.

John Donne

A la suite de Cicéron, les Elisabéthains établissent, dans leur théorie critique, une ferme distinction entre historia et fabula, ${ }^{1}$ entre le véridique et le merveilleux, auquel se rattache le mythique, opposition qui se retouve dans la comparaison qu'établit Sidney entre histoire et poésie. ${ }^{2}$ La vision qu'ils ont de l'histoire est pourtant le lieu privilégié d'une création mythique. Au temps de la Renaissance, certains mythes de civilisation, souvent combinés à une interprétation évhémériste des dieux de l'Antiquité, montrent l'homme primitif dénué de tout art et apprenant peu à peu, sous l'influence de héros civilisateurs, à construire et organiser une communauté. A l'opposé, sèmble-t-il, le mythe de l'âge d'or implique un déclin continu depuis un état primitif de bonheur innocent jusqu'aux désordres incessants de l'âge de fer. Mais l'âge de fer doit être suivi d'un nouvel âge d'or dans une conception cyclique de l'histoire, tandis que les mythes progressistes n'excluent pas un développement non linéaire; dans la Méthode de l'histoire, Jean Bodin considère que l'humanité progresse de l'état sauvage à l'état civilisé, mais que ses progrès sont suivis de rechutes. Car

La nature semble soumise à la loi de l'éternel retour, ou chaque chose est l'objet d'une révolution circulaire de sorte que le vice succè de à la vertu, l'ignorance à la science, le mal à l'honnéteté, les ténè bres à l'erreur ${ }^{3}$ 
Ainsi les deux mythes, de construction et de dégradation, peuvent se trouver inclus dans un mythe de naissance, développement, déclin, mort et renaissance, que renforce l'homologie familière entre la société, le corps humain, et le passage des saisons. ${ }^{4}$

La synthèse qu'implique le mythe de renaissance cherche à s'établir sous le règne d'Elizabeth, dans un équilibre infiniment complexe, non exempt de tensions et de tiraillements souterrains, et dont seuls quelques aspects pourront être somm airement abordés ici.

L'une de ses expressions les plus claires est peut-être fournie par Spenser dans The Faery Queene. Le début du livre V décrit la détérioration du monde. De l'âge d'or, il est parvenu à l'âge de pierre, comme l'exprime le mythe de Deucalion, qui ressuscita le genre humain en lançant des pierres par dessus son épaule; ce sont ces pierres qui tiennent lieu de coeur à tous les membres de la race nouvelle. ${ }^{5}$ Un mythe de renaissance est ainsi transformé en mythe de dégénérescence. Mais le propos du moraliste n'est pas en contradiction avec celui de l'apologiste de la civilisation élisabéthaine qui développe le mythe de la renaissance, en la personne d'Elizabeth, de la lignée troyenne issue des dieux :

Of the antique Troian stocke, there grew Another plant, that raught to wondrous hight, And far abroad his mighty branches threw... ${ }^{6}$

Tandis que l'annonce de l'avènement d'un nouvel âge d'or indique souvent le désir d'échapper à un sombre présent, ${ }^{7}$ la conscience de l'indignité des hommes, de leur déchéance par rapport à un paradis perdu, doit les inciter à opérer en eux-mêmes une métamorphose qui leur fasse aimer la justice d'une Astrea revenue et permette leur propre renaissance. Le mythe troyen, que les historiens élisabéthains discutent parfois, mais acceptent pour la plupart ${ }^{8}$ - si bien que les attaques de Thomas Fenne demeurent isolées ${ }^{9}-$ constitue chez Spenser une vision historique selon laquelle une période qui se veut brillante établit 
une continuité avec une ère glorieuse par delà les temps d'obscurité, de déclin et d'oubli. Le phénomène est répétitif dans la vision de Merlin, qui annonce qu'une nouvelle lignée

Shall spring, out of the auncient Troian blood, Which shall reuive the sleeping memorie

Of those same antique Peres, the heauens brood, Which Greeke and Asian riuers stained with their blood (III. iii. 22),

utilisant la même métaphore végétale que le fantôme de Gorlois, qui prédit, à la fin de The Misfortunes of Arthur, l'avenement de «That vertuous Virgo ... // That pierelesse braunch of Brute . . .// that hope of springing Troy» (V. ii. 18-20), ou que Bacon dans les derniers vers de Friar Bacon and Friar Bungay. ${ }^{10}$ De renaissance en déchéance, le fils est incapable de conserver ce qu'a fondé le père, jusqu'à ce que le petit-fils renouvelle "the old sparkes...// Of natiue courage» (Faery Queene, III. iii. 45). L'image est reprise et développée pour annoncer le règne d'Henry VII :

... when the terme is full accomplishid, There shall a sparke of fire, which hath long-while Bene in his ashes raked up, and hid, Be freshly kindled in the fruitfull lle Of Mona...

(Ibid., III. iii. 48) ${ }^{11}$

Cette métaphore des cendres d'où ressurgit la flamme est exactement homologue de l'image du phénix, souvent appliquée à la renaissance de Troie ${ }^{12}$ ou à Elizabeth, en qui revivent les grands fondateurs troyens. ${ }^{13}$ Or en méme temps, le chant ix du troisième livre, qui est l'un des lieux, dans The Faery Queene, où est annoncée la renaissance de Troie en Grande Bretagne, constitue en lui même une autre renaissance. Tandis que Britomart 
raconte comment Troie fut reconstruite en Rome et le sera une troisième fois en Londres, Paridell et Hellenore échangent des regards amoureux comme l'avaient déjà fait ceux dont leur nom est dérivé, Pâris et Hélène, si bien que nul ne peut s'étonner, au chant suivant, de lire l'enlèvement de Hellenore par Paridell. Le texte, de façon explicite, en nommant "This second Hellene, faire Dame Hellenore» (III. x. 13), fait renaître dans la mémoire un texte plus ancien. De méme, lorsque, au livre III, Merlin fait apparâ̂tre à Britomart la glorieuse descendance troyenne, le texte se fait recréation de celui de l'Arioste, où, dans le chant III, devant le tombeau de Merlin, Melissa découvre à Bradamante sa brillante lignée (III. 17-48). The Faery Queene participe d'un projet avoué de renaissance littéraire. Au livre IV, le poète écrit une suite au Conte du Squire; c'est que le temps a en partie détruit l'oeuvre de Chaucer, qu'il va tenter de faire revivre

through infusion sweete Of thine owne spirit, which doth in me surviue.

(IV. ii. 34)

Le poème, dans sa totalité, jette un double regard en arrière aux épopées de l'Arioste et de Virgile, inscrivant dans son texte la double résurrection de la grandeur politique et de la pratique littéraire. Les premiers vers de The Faery Queene, qui annoncent le retour à la vie, après un long sommeil, des nobles dames et chevaliers, rappellent en même temps, par allusion et imitation, le début de l'Enéide, ${ }^{14}$ qui est le poème de la renaissance de Troie en Rome, et la reine Elizabeth, nouvel Auguste aussi bien que nouvel Enée, se confond également avec la muse inspiratrice, par l'opération d'une intertextualité métaphorique typique de la Renaissance, en rupture avec l'intertextualité métonymique médiévale. ${ }^{15}$ L'identité de la destinée des Etats et de celle des arts est nettement marquée par Puttenham dans The Arte of English Poesie ${ }^{16}$ et s'il part en guerre contre le mépris dans lequel sont tombés les poètes de son temps, ${ }^{17}$ c'est pour mieux créer les conditions d'un renou- 
veau dont il affirme avec force la possibilité, ${ }^{18}$ comme aussi Alexander Nevyle dans la préface à sa traduction anglaise de l'Oedipe de Sénèque, ${ }^{19}$ renouveau qui consiste aussi à restaurer, pour Spenser, «as to theyr rightfull heritage such good and naturall English words, as haue ben long time out of use and almost cleane disherited», ${ }^{20}$ recréation chaque fois indissociable, dans l'esprit du créateur, d'un propos śthique. Car le mythe de renaissance semble exprimer dans l'idéologie élisabéthaine une isomorphie fondamentale entre la création de l'Etats, l'élaboration et la consolidation d'une éthique, et la création artistique. Comme dans la Renaissance continentale, il s'agit, selon l'expression de Chastel, d'un

effort de plus en plus manifeste pour concevoir l'éthique, les affaires publiques, le savoir, l'art, comme les diverses faces d'une même réalité centrale, donc [. . .] un travail d'integratio, de vision globale, qui ne peut lui-même être dissocié d'une volonté de possession et de dépassement ou renovatio, à la résonance complexe, qui rejette toujours vers l'avenir. ${ }^{21}$

Lorsqu'il met en scène le choix de Pâris, en attribuant la pomme d'or à Zabeta, ${ }^{2} 2$ non seulement Peele ressuscite un texte, un mythe ancien, mais le transforme de manière à unir la fiction et l'histoire, et à métamorphoser la rivalité entre Junon, Minerve et Vénus, la division entre le pouvoir, la connaissance, la beauté, en une indissociable unité. La nouvelle Troie, ainsi, ne sera pas détruite. A travers le mythe de la renaissance de Troie, du phénix, ou de la végétation, s'exprime une vision de l'histoire semblable à celle que Pétrarque avait suscitée en Italie deux siècles auparávant. ${ }^{23}$ L'histoire même, conçue comme renaissance, devient un réservoir de modèles pour l'action présente; et la rhétorique des comparaisons mythologiques, encomiastiques ou non, est à situer dans $œ$ contexte. Les nécessités du présent font aussi que l'on se tourne volontiers vers le passé pour y trouver la justification des solutions proposées. Comme le 
montre, par exemple, l'étude récente de Ferguson sur la perception du passé social et culturel dans l'Angleterre du XVIème siècle, l'exploration historique - non comme récit de res gestae, mais comme étude de société-commence à se développer par le biais, entre autres, des recherches déclenchées par la Réforme sur la relativité temporelle des institutions ecclésiastiques. ${ }^{24}$ Le regard en arrière est indissociable d'une projection dans l'avenir. Il est tout significatif que dans leurs poèmes en l'honneur de Chapman et de sa traduction des Travaux et des Jours d'Hésiode, tandis que Drayton parle de la redécouverte d'un trésor perdu,

Thou hast vnlock'd the treasury, wherein

All Art, and knowledge haue so long been hidden, 25

Ben Jonson associe intimement la renaissance du passé avec la découverte de continents nouveaux :

If all the vulgar Tongues that speake this day, Were askt of thy Discoueries; They must say, To the Greeke coast thine onely knew the way. Such Passage hast thou found, such returns made ... 26

Mais la synthèse porte en elle le germe de son propre éclatement. Si le mythe de renaissance a donné une puissante impulsion au progrès des études historiques et fait naître le sens de l'histoire, ${ }^{27}$ le développement d'une science historique voulue objective devait contribuer à scinder mythe et histoire, et à tuer le mythe.

Des dangers de division apparaissent nettement lors du passage du poème épique à la forme théâtrale. L'organisation d'ensemble des tétralogies shakespeariennes relève d'une vision historique inspirée du mythe. Henry V comme Henry VII instaurent le renouveau. A la fin d'Henry VIII est annoncé le règne du phénix qui, à la mort d'Elizabeth renaitra sous les traits de Jacques ler. ${ }^{28}$ Mais les forces 
contraires sont puissantes. La première partie d'Henry VI s'ouvre sur une atmosphère de désenchantement et d'inquiétude :

«Henry is dead and never shall revive».

La première scène place la pièce sous le signe de la mort et de l'impossibilité de renaissance à plusieurs reprises énoncée ${ }^{29}$ et plus tard développée grâce à la métaphore végétale resémantisant celle de l'arbre généalogique. Les jardins d'Adonis, symbole, chez Spenser, de la fécondité universelle, ne sont ici que dérisoire image de succès fragile, éphémère. ${ }^{30}$ De même que le jeune Henry VI est incapable de faire revivre en lui le roi défunt, le fils de Talbot, qui devait perpétuer le renom de son père, ${ }^{31}$ meurt avec lui prématurément. L'arbre, la fleur, la vigne cherchent la croissance et l'épanouissement, ${ }^{32}$ mais inexorablement flétrissent, ${ }^{3} 3$ et les pousses nouvelles sont brisées, arrachées, rongées par les insectes, pourries par la tempête; ${ }^{34}$ le bûcheron s'acharne contre le chêne, ses rejetons et ses racines. ${ }^{35}$ Toujours demeure, cependant, l'espoir d'une résurrection ; Lucy fait emporter les cadavres des deux Talbot,

But from their ashes shall be rear'd

A phoenix that shall make all France afeard.

( I Hen. VI, IV. vii. 92-93)

Les insultes et les tortures dont la reine Margaret tourmente le duc d'York rappellent les moqueries adressées au "roi des Juifs» lors de son supplice; York lui-même reprend un autre symbole christique :

My ashes, like the phoenix, may bring forth

A bird that will revenge upon you all.

(3 Hen. VI, I. iv. 35-36),

mais la résurection, dans un enchafnement fatal, se met 
ici au service de la vengeance et de la mort.

Métaphoriquement, des forêts entières sont déracinées, ravagées par le feu. La victoire sur la foret ${ }^{36}$ emprunte dans le discours de Gloucester ou d'Edouard l'apparence trompeuse de l'oeuvre civilisatrice de défrichement, qui ne saurait masquer ici l'oeuvre réelle de destruction. Le premier deviendra vite «rooting hog», ${ }^{37}$ «The wretched, bloody, and usurping boar, // That spoil'd your summer fields and fruitful vines». ${ }^{38}$

Dans l'alternance d'exils et de retours à nouveau suivis de bannissements «from Princely bowre to wastefull wood», ${ }^{39}$ les tétralogies montrent dans la dimension temporelle, historique, la confrontation entre les forces de destruction et de civilisation, de division et de renouveau que la pastorale organise dans l'espace. Entre les deux types de vision, l'articulation se fait au moyen de la métaphore végétale et sans doute faudrait-il considérer sous cet angle la réflexion d'Henry VI pendant la bataille de Tow ton ( $3 \mathrm{Hen}$. $V I$, II. v.), dans un monologue qui non seulement oppose la vie du berger et celle du roi, mais associe à la vie pastorale le déroulement cyclique des saisons, alors que sous ses yeux, le renouvellement des générations humaines est symboliquement mis en cause dans le combat du fils qui tue son père et du père qui tue son fils.

$\mathrm{Si}$ la renaissance, comme dans The Faery Queene, est perçue comme réunion et synthèse, la décadence est le fruit logique d'une scission, d'un écartèlement. Des oeuvres comme Gorboduc, The Misfortunes of Arthur, Locrine et encore King Lear, mettent en scène le déclin suscité par la division du royaume, image de la faute initiale, vrai péché originel, qu'avait été la partition du territoire de l'ancêtre troyen Brut entre ses héritiers. La disjonction que symbolise Henry VI entre la vie contemplative et la vie active conduit à l'impuissance. Inversement, comme le comte de Richmond réconcilie les deux roses, Henry $V$ réunit en lui-meme l'imagination théâtrale et l'efficacité de l'action, qui s'étaient opposées dans l'affrontement entre Richard II et Bolingbroke.

Le vieux roi Henry IV meurt, tué par une vision 
des choses pessimiste et partielle. La philosophie de l'histoire qu'exprime Warwick,

There is a history in all men's lives

Figuring the nature of the times deceas'd;

The which observ'd, a man may prophesy,

With a near aim, of the main chance of things

As yet not come to life, who in their seeds

And weak beginnings lie intreasured.

Such things become the hatch and brood of time

(2 Hen. IV, III. i. 80-86),

voit l'avenir contenu en germe dans le passé. Loin de percevoir là une chance, grâce à une connaissance de l'ancien, de maîtriser et modeler le nouveau, le roi ne voit que le poids du déterminisme : "Are these things then necessities ?» (ibid., 92). Il est ainsi condamné à ne rien comprendre à l'entreprise de renaissanœ menée par son fils, qu' il perçoit comme un retour du royaume aux temps de la sauvagerie préhistorique, quand la forêt recouvrait tout le territoire :

O thou wilt be a wilderness again, Peopled with wolves, thy old inhabitants

(ibid., IV. v. 136-37)

C'est qu'à ses yeux, le prince Henry ressuscite Richard II dans sa trop grande familiarité avec le peuple, qui «surfeited with honey, and began // To loathe the taste of sweetness》 (1 Hen. IV, III. ii. 71-72). Il est vrai que le miel semble se dégrader à tel point qu'il devient-méme si ce n'est que par antiphrase - attribut de Mistress Quickly, douce «as the honey of Hybla» (ibid., I. ii. 41) et que celle-ci inverse toutes les valeurs lorsque, dans une confusion révélatrice, homicidal se mue en honey seed ; le meurtre prend l'apparence de l'union du miel et de la fécondité ( $2 \mathrm{Hen}$. IV, II. i. 50). Si bien qu'il peut paraître inquiétant que le prince devienne, pour ses compagnons de débauche, «my good sweet honey lord» (l Hen. IV , I. ii. 158). Comme le pense le roi, 
Tis seldom when the bee doth leave her comb In the dead carrion

(2 Hen. IV, IV. iv. 79-80)

L'image rappelle l'épisode du livre des Juges où Samson, après avoir tué un lion,

se détourna pour voir le cadavre du lion : voici qu'il y avait dans la carcasse du lion un essaim d'abeilles et du miel. ${ }^{40}$

Il y a pour Henry IV incompatibilité entre la pourritureles mauvais compagnons, la déchéance, éventuellement la mort - et le miel de la parfaite éducation princière et de la prospérité de l'état. Ce que dément l'exégèse commune, telle que la résume Valeriano dans les Hieroglyphiques :

Cela se peult peindre, quand quelqu'vn chargé de mesdisances \& calumnies, en obtient vn surcroist de reputation, ou quand les outrages \& contumelies de ses malueillans ne font que redoubler la gloire de ses merites. Car le lion est extrêmement dangereux de la dent, plein de violence \& ferocité. Et les Abeilles qui font leur miel dedans sa gueule, monstrent la douceur $\&$ prosperité qui suit apres l'affliction.

L'idée est clairement exprimée par le prince :

Yet herein will I imitate the sun, Who doth permit the base contagious clouds To smother up his beauty from the world, That, when he please again to be himself, Being wanted he may be more wonder'd at By breaking through the foul and ugly mists Of vapours that did seem to strangle him (1 Hen. IV, I. ii. 192-98), et sera reprise dans Henry $V$ en termes végétaux : le prince 
apparaît alors avoir couvert sa sagesse d'un manteau de folie,

As gardeners do with ordure hide those roots That shall first spring and be most delicate

(Hen. V, II. iv. 38-39) ${ }^{42}$

Il y a la même opposition apparente entre lumière et ténèbres, fleur et fumier, miel et pourriture. Et la même relation intime : comme les ténèbres rendent plus vive la lumière, le miel est d'autant plus suave qu'il est recueilli en un lieu répugnant. En fait, la relation est plus profonde, et plus fondamentale : la fleur doit son existence même au fumier. Semblablement, dans la vision héroïque du roi à la veille d'Agincourt, où il compte «gather honey from the weed» (Hen. V, IV. i. 11), de la carcasse des morts anglais enterrés dans les fumiers français, le soleil fera monter jusqu'au ciel une vapeur glorieuse (ibid., IV. iii. 98-101).

Dans le livre IV des Géorgiques de Virgile, Aristée,le berger d'abeilles, qui avait indirectement causé la mort d'Eurydice, en fut puni par la perte de tous ses essaims. Pour apaiser la colère des dieux, il dut sacrifier quatre taureaux et autant de génisses. Et voici qu'à la neuvième aurore

on vit se produire un prodige soudain et merveilleux : au milieu des viscères liquéfiés des boeufs, bourdonnent des abeilles : elles s'échappent des flancs et des côtes rompues, forment d'immenses traînées de nuages, volent en masse au sommet d'un arbre et laissent pendre leur grappe de ses flexibles rameaux. ${ }^{43}$

Une interprétation du phénomène était fournie à la Renaissance par le quinzième livre des Métamorphoses d'Ovide, dans l'exposé de la doctrine de Py thagore. Ce qui est miraculeux chez Virgile appartient ici au naturel :

choisissez une fosse, immolez-y des taureaux et rejetez sur eux de la terre; par un phénomène 
que l'expérience atteste, de leurs chairs en putréfaction naissent çà et là des abeilles qui vont butiner les fleurs.

Cette image de résurrection figure, dans les Métamorphoses, à côté de deux autres, dont elle est l'homologue, celle du phénix (Mét., XV. 391-407), et celle de Troie (ibid., 424, 431-52). N'est-ce pas Melissa - l'abeille - qui montre à Bradamante, dans le Roland Furieux, la résurrection de la lignée troyenne?

Dans la seconde tétralogie, le miel est le produit idéal de la communauté civile parfaite que représente traditionnellement la ruche, comme dans le discours de l'Archevêque de Cantorbéry (Hen. V, I. ii. 187-203). ${ }^{45}$ Le miel est alors aussi la paix qui naît de la guerre, comme dans l'emblème de la ruche nichée en un casque, emblème d'Alciat ${ }^{46}$ repris par Whitney, ${ }^{47}$ et par Lyly dans Euphues ${ }^{48}$ pour caractériser la paix élisabéthaine, plus profonde que celle du règne de Numa Pompilius ; ou comme dans l' $\mathrm{i}$ mage du lion de la gueule duquel sortaient des abeilles lors de la remise de l'Ordre de la Jarretière à Henry IV en 1596 à Rouen, enjoignant au roi de

$$
\begin{aligned}
& \text { rule and govern prosperously } / / \text { Your people } \\
& \text { both in warre and peacefull sort }, 49
\end{aligned}
$$

image proche de la devise usuelle «De forti dulcedo». ${ }^{50}$ $\mathrm{La}$ douceur n'est issue que de la force, et la guerre ne doit avoir lieu que pour établir la paix. Le vrai prince civilisateur est ainsi producteur et protecteur du miel, image d'un haut degré de culture - qu'il n'est souvent possible d'atteindre qu'après une traversée du désert, comme lorsque les Hébreux découvrirent la terre de lait et de miel (Exode, 3, 8) - ou qui met un terme à des temps plus barbares : c'est ainsi qu'Aristée, maître du miel, fit passer l'humanité de l'ère de la viande, de la chasse et de la boucherie, à l'ère pastorale du miel, comme l'expliquent Natale Conti, ${ }^{51}$ Valeriano, ${ }^{52}$ Pierre Dinet ${ }^{53}$ et comme le montrent aussi deux tableaux de Piero di Cosimo; ${ }^{54}$ étape dans le retour 
vers l'âge d'or, où «l'yeuse au vert feuillage distillait le miel blond». 55 C'est la tâche entreprise par le futur Henry V, de faire naitre les abeilles de la charogne.

Dans une interprétation plus large du mythe le miel figure, en même temps que l'édification d'une société, l'élaboration d'une culture symbolisée le plus souvent par la création artistique. Les abeilles sont les oiseaux des Muses, dit LyLy dans Euphues, toute fausse note les affole. ${ }^{56}$ La métaphore d'Horace et de Sénèque, qui fait du poète une abeille butinant diverses fleurs pour produire son miel, est au centre de la théorie de l'imitation créatrice de la Renaissance. ${ }^{57}$ Plus spécifiquement, la rosée des Muses figure l'éloquence. ${ }^{58}$ Non seulement Henry $\mathrm{V}$ surprend par sa nouvelle sagesse - «Never was such a sudden scholar made» (I. i. 32) -, mais subjugue par le pouvoir de son éloquence, «his sweet and honeyed sentences» (I. i. 51). Mais alors que dans l'idéal spensérien la créativité artistique, l'art politique et moral sont indissociables, modalités d'un seul acte créateur, on voit dans la seconde tétralogie s'opérer un glissement. Tandis que Richard II, en bon acteur, joue sa propre destitution, Henry $\mathrm{V}$ assoit sa légitimité en organisant, dans une habile mise en scène, sa propre renaissance. La scène où le prince et Falstaff jouent comme au théâtre l'entretien entre Henry IV et son fils ( 1 Hen. IV, II. iv. 371475 ) souligne la nature théâtrale de l'entreprise de renaissance. Si l'action politique reste informée par une créativité d'ordre artistique, celle-ci non seulement lui est subordonnée et seconde, mais menace de l'entraîner dans le domaine ambigu de l'apparence ; indice, alors même que s'affirme, sous forme de synthèse, une renaissance victorieuse, que les forces de disjonction demeurent à l'oeuvre.

Elles deviennent prédominantes dans la tragédie de Hamlet, qui, observée sous cet angle, semble inverser les structures de la deuxième tétralogie. Si le prince y couvre aussi sa sagesse d'une apparence déraisonnable, ce n'est pas qu'un dévoilement se prépare ; au contraire, les distinctions bientôt se brouillent entre le masque et le visage. 
L'action prend la forme, métaphoriquement et littéralement, d'une mise en scène, mais pour souligner le plus souvent un divorce entre l'imagination et l'acte, ou entre "The courtier's, soldier's, scholar's, eye, tongue, sword» (III. i. 153). L'adéquation entre la fiction - douleur d'Hécube - et la représentation - larmes de l'acteur - ne rend que plus éclatante, dans un déchirement de l'être, la disparité entre le verbe et l'acte. Le regard vers le passé ne produit qu'un sentiment de faiblesse, de dégénérescence entre Hypérion et le satyre (1. ii. 140), entre Hercule et Hamlet (ibid., 153). Dès lors la chute de Troie, dans la tirade du «Premier acteur», loin d'annoncer la renaissance habituelle, est emblème tragique de la fin d'un monde. Les thèmes de renaissance, les métempsycoses py thagoriciennes, se colorent d'ironie sarcastique ou amère : le soleil ne fait plus naître que des asticots dans la carcasse des chiens (II. ii. 182), le cycle de la vie n'est qu'une constante dégradation de l'homme au ver qui s'en nourrit (IV. iii. 21-31), ou du corps d'Alexandre au bouchon de glaise qui obture une barrique (V. i. 196-209). L'exhumation des squelettes rompus emblématise l'impossibilité de renaissance; et lorsque Laertes souhaite que du corps d'Ophélie naissent des violettes (V. i. 233-34), reviennent à l'esprit ses propres paroles, qui avaient fait de cette fleur un symbole de précarité,

Forward, not permanent, sweet, not lasting, The perfume and suppliance of a minute,

No more (I. iii. 8-10)

La terre entière n'est-elle point devenue «promontoire stérile» (II. ii. 299) ?

Peut-être le tragique exprime-il les angoisses d'une époque, ou ce qui contrarie ses idéaux profonds. Toujours est-il que l'impossibilité de renaissance - liée à une division, est un des thèmes profonds du tragique shakespearien. Déjà chez Kyd, dans The Spanish Tragedy, l'assassinat d'Horatio est symboliquement perpétré dans une tonnelle verdoyante et le cadavre pendu, par dérision, comme fruit sanglant de l'amour. L'une des scènes emblématiques de la 
pièce (IV. ii) montre la destruction de la végétation par la mère d'Horatio, figure de fécondité détruisant la fertilité. Retiré dans le désert, Timon d'Athènes sera prêt à couper le même arbre emblématique (Timon, V. i. 20410). Dans Macbeth, le martinet accroche son berceau fécond aux flancs du château du meurtre, et l'on cherche à détruire les enfants, symboles de renouveau; ${ }^{59}$ les branches de verdure, ambivalentes, camouflent l'armée, force destructice qui proclame son pouvoir de renouvellement, car il semble falloir que la forêt recouvre, comme aux ori gines pré-culturelles, le pays qui a perdu sa civilisation, afin que de ses profondeurs obscures surgisse le rameau d'où naîtra la longue lignée de rois civilisateurs issus de Banquo. La forêt sauvage (silvatica) est matrice de civilisation. Artegall était apparu à Britomart symboliquement déguisé en homme des bois «With woody mosse bedight» (The Faery Queene, IV, iv. 39), de même qu'est fils de la forêt Silvius, l'ancêtre troyen. Comme Macbeth, au plus profond de la tragédie, met en danger «the treasure // Of Nature's germens» (Macb., IV. i. 58-59), Lear ne trouve malédiction plus absolue que d'en appeler à la destruction universelle, à l'arrêt du cycle de renaissance :

Crack Nature's moulds, all germens spill at once That makes ingrateful man!

$$
\text { (Lear, III. ii. 8-9) }
$$

C'est le méme cauchemar d'impossible renaissance qu'évoque Donne dans An Anatomy of the World:

Spring-times were common cradles, but are

tombs;

And false conceptions fill the general wombs 60

Lear avait appelé sur Goneril la stérilité (Lear, 1. iv. 284-98), car celles qui sont issues de lui, et en qui il devrait renaitre, en s'arrachant à leur racine se vouent à la mort :

She that herself will sliver and disbranch 
From her material sap, perforce must wither And come to deadly use (Lear, IV. ii. 34-36)

L'impossibilité de résurgence ici encore a pour cause la division du royaume, la division entre le père et l'enfant, le frère et le frère, la soeur et la soeur, le roi et le sujet, l'homme et les dieux, la division, enfin, de l'etre - ruptures rappelées par les images d'écartèlement, de brisure, de déchirement, et généralement de torture physique si fréquentes dans cette pièce, qui offre une image de fin du monde (ibid., V. iii. 263).

Sans doute est-il inutile de rappeler combien est central dans les «Romances» le thème de renaissance, que l'on peut symboliser par les fleurs de Perdita ou l'emblème de Pericles, «A wither'd branch, that's only green at top» (Per., II. ii. 42). La résurrection n'y est pas miraculeuse, mais s'entoure d'une atmosphère étrange et mystérieuse qui estompe les frontières entre le naturel et le merveilleux. Surprenante magie, la scène de la résurrection de Thaisa, la transmutation d'Alonso dans la chanson d'Ariel, le réveil de la statue d'Hermione; des secrètes profondeurs du rêve parvient à Posthumus l'énigme fondamentale :

from a stately cedar shall be lopp'd branches, which, being dead many years, shall after revive, be jointed to the old stock, and freshly grow (Cymb., V. iv. 140-43) ${ }^{61}$

Les renaissances, à tous les niveaux, mettent un terme aux divisions et dispersions initiales. Elles n'ont jamais été aussi profondes, mais jamais non plus aussi pénétrées de la fragilité de l'impalpable. Au moment où le théâtre, conscient de son immence puissance de résurrection ${ }^{62}$ - qu'il doit à une illusion suffisamment forte pour que Cléopâtre ait pu, sans la mettre en danger, souligner ce qu'a de précaire sa propre renaissance (Ant. \& Cleo., V. ii. 215-20) - est conscient aussi d'être fait de visions évanescentes qui se fondent «into air, into thin air» (Temp., IV. i. 150), les «Romances» associent constamment renaissance, réveil, 
retour, régénération au sens aigu de l'illusoire, si bien que le phénix, dans la Tempéte, est devenu emblème de l'incroyable (III. iii. 21-24). En insistant sur leur caractère fabuleux, les «Romances» orientent l'oeuvre de Shakespeare de l'historia vers la fabula ${ }^{63}$ et soulignent l'irréalité du conte pour mieux fondęr la vérité du mythe. Si elles ont cependant une conscience historique, c'est dans l'emploi de techniques théatrales délibérément désignées comme conventionnelles ou archaïques. ${ }^{64}$ Le vieux Gower renait de ses cendres ; le Temps lui-même, en vers démodés, sert de choeur au Conte d'hiver. Est-ce un moyen d'exprimer l'intemporalité du mythe, au moment même où la pensée mythique commence à ne plus être reconnue comme outil de la connaissance, tandis que, dans le domaine de l'expression litteraire, Thomas Carew se plaît à louer Donne d'avoir banni de ses meilleurs vers «the goodly exil'd traine // Of gods and goddesses» ${ }^{65}$ et que Heywood, dans la série des Quatre Ages historicise la my thologie ? La conscience de la dissolution d'un monde s'allie-t-elle à l'assurance que la véritable renaissance se trouve, comme le pressentait déjà le roi Lear, dans les «vieilles légendes»et les «papillons dorés» (Lear, V. iii. 12-13), dans la puissance et la permanence de l'imaginaire?

Un élément de réponse est peut-être fourni par l'histoire d'Orphée. Dans le regard d'Orphée, selon Blanchot, ${ }^{66}$ s'inscrit l'inspiration. L'oeuvre d'Orphée est de ramener au jour le centre de la nuit, qu'il ne peut jamais voir en face. Se retourner vers Eurydice détruit l'oeuvre, mais il est nécessaire qu'Eurydice soit perdue pour que le chant existe.

En se retournant en arrière, la Renaissance approfondit sa conscience historique, mais, comme le remarque Delumeau,

Interposant l'épaisseur des «temps obscurs» entre l'Antiquité et le nouvel âge d'or, elle rejeta définitivement dans le passé, comme quelque chose de révolu, une civilisation dont elle désirait s'inspirer, mais qu'il lui était impossible de ressusciter. 67 
Le rêve de synthèse se fonde ainsi sur une rupture. Aussi, le regard en arrière des Elisabéthains, d'où naît le renouveau, cherche à construire un idéal qui ne s'accomplit peut-être totalement que dans la conscience de son échec. Orphée représente le poète civilisateur capable de réaliser un nouvel âge d'or. ${ }^{68}$ Tel l'ont décrit, à la suite de Boccace, de Natale Conti et d'Arthur Golding, Richard Wills, Thomas Lodge, William Webbe, Puttenham et Sidney dans leurs arts poétiques. ${ }^{69}$ La poésie ne se différencie alors aucunement de la philosophie, de la connaissance et de l'action, mais les contient toutes. A trois reprises ${ }^{70}$ Spenser fait allusion à la descente d'Orphée aux enfers en n'en montrant que l'épisode victorieux ou même en transformant la fable de manière à lui donner une issue heureuse. L'inspiration poétique est une force d'amour qui, seule forme supérieure de l'art, permet d'échapper aux «ruines du temps» et peut-être, comme l'union du phénix et de la tourterelle, de fusionner le multiple dans l'un.

Mais la Renaissance, époque des grandes synthèses et de la discordia concors, est aussi celle des grandes divisions, politiques, religieuses, intellectuelles. Machiavel dissocie l'éthique et la politique, et son réalisme rigoureux

réduit le déroulement des événements humains à une succession désordonnée et sans but, à un retour cyclique qui fait de l'aventure de l'homme sur terre un «conte» inutile et vain. 71

Les sciences se constituent en s'entourant de frontières et la recherche de l'unité philosophique par intégration ${ }^{72}$ commence. à céder le pas à l'établissement d'unités de connaissance par exclusion. C'est alors que Donne, déplorant la dégénérescence de la race humaine, ${ }^{73}$ reprend les termes mêmes de Hamlet :

Then, as mankind, so is the world's whole frame Quite out of joint. ${ }^{74}$

De ce monde «all in pieces, all coherence gone» ${ }^{75}$ on ne 
peut faire que la «dissection». Le phénix, qui ailleurs, comme l'a montré Jean-Marie Maguin, 76 figure la renaissance dans l'union des amants ou dans l'amour divin, n'est plus ici symbole de renouvellement, mais de l'unicité où s'enferme chacun, toute relation rompue :

Prince, subject, father, son, are things forgot, For every man alone thinks he hath got

To be a phoenix, and that then can be

None of that kind, of which he is, but he. 77

Et méme si une résurrection est finalement envisagée, elle est individuelle, et sous forme de séparation tripartite, la tombe gardant le corps, Dieu l'âme, le poète la renommée. ${ }^{78}$

$\mathrm{Au}$ moment où la poésie et la philosophie, le my the et l'histoire, la vision artistique et la vision rationaliste de l'univers sont en train de se scinder, le rêve d'idéale identité entre l'art et la vie s'effondre. Alors les poètes anglais oublient le triomphe d'Orphée, pour ne plus guère chanter que le démembrement final et la rage des Bacchantes. ${ }^{79}$ Dans The Wisdom of the Ancients, Francis Bacon voit en Orphée une figure de la philosophie, qui apprend aux hommes à vivre en communautés harmonieuses. Mais les civilisations déclinent, Orphée meurt écartelé,

learning and philosophy must be dismembred, so that a few fragments onely, and in some places will bee found like the scattered boords of shipwracke. ${ }^{80}$

Tandis que s'aiguise la conscience de ces divisions, Shakespeare montre en Prospero celui qui dans son duché avait séparé la connaissance de l'action ; mais sur l'île enchantée, la vie, l'art et le mythe se confondent si bien que la vie acquiert le caractère illusoire de l'art et du fabuleux. Peutêtre est-ce au moment même où le my the comprend qu'il est un mythe, où le rêve se découvre tel, qu'il atteint, dans sa mort, sa plus haute perfection. 
Certes, Orphée fut déchiré, lacéré, ses membres épars dispersés dans l'Hèbre. Mais sa tête arrachée, portée par les flots vers un lointain rivage, vint doucement s'y échouer, et là, se remit à chanter... 
NOTES

1. De Inventione, I. xix.

2. A Defence of Poetry, éd. J. A. Van Dorsten, Oxford U. P., $1966(1982)$, pp. $35-38$.

3. J. Bodin, éd. P. Mesnard, t. v, 3 du Corpus général des philosophes français, Paris, 1951 , pp. 428-29, cité par J. Delumeau, Le péché et la peur. La culpabilisation en Occident XIIIè-XVIIIè siècles, Paris, 1983 , p. 140 .

4. Le cycle complet se trouve exposé par Lucrèce.: voir Erwin Panofsky, Studies in Iconology. Humanistic Themes in the Art of the Renaissance, Oxford, 1939 (New York, 1972), p. 40, n. 22. Triom phante, la Renaissance voit surtout les premiers temps du cycle; en crise, elle insiste, comme Donne dans An Anatomy of the World, sur la phase de déclin.

5. The Faery Queene, V, proem, $1-2$; Spenser s'inspire de Natale Conti, Mythologiae, VIII. xvii, pp. 896-901 (nous utilisons l'éd. de Francfort, A. Wechel, 1584).

6. The Faery Queene, III. ix. 47.

7. Delumeau, op. cit., p. 138.

8. Voir Douglas Bush, Mythology and the Renaissance Tradition in English Poetry, New York, 1932 (1960), pp. 3740 ; A. E. Parsons, "The Trojan Legend in England, Some Instances of its Application to the Politics of the Time», Modern Language Review, XXIV (1929),pp. 253-64 et 394-408. Même William Camden, qui rapporte toutes les attaques qui ont été dirigées contre cette histoire, $n$ 'en conclut pas moins que "For mine owne part, it is not my intent, I assure you, to discredit and confute that storie which goes of him, for the upholding whereof, (I call Truth to record) I have from time to time streined to the heighth, all that little wit of mine. For that were, to strive with the streame and currant of Time; and to a struggle against an opinion commonly and long since received", Britain, or a Chorographical Description of the most flourishing Kingdomes, England, Scotland, and Ireland, and the Ilands adicyning, out of the depth of Antiquitie. Written first in Latine by William Camden. Translated newly into English by Philemon Holland, Londres, 1610 , p. 6 (1 ère éd., 1586).

9. Sur Fennes Frutes (1590), voir Mark Lewis Richardson, The Legends of Troy in the English Renaissance: A Study in Decadent Literature, thèse dactylographiée, Emory University, 1980 , pp. $42-53$.

10. «I find by deep prescience of mine art, $/ / \ldots / /$ That here where Brute did build his Troynovant, // From forth the royal garden of a king // Shall flourish out so rich and fair a bud // Whose bright. ness shall deface proud Phoebus' flower, // And overshadow Albion 
with her leaves» (xvi, $42-48)$.

11. Voir aussi ibid., III. ix. 38 : "For noble Britons sprong from Troians bold, // And Troynouant was built of old Troyes ashes cold».

12. P. ex, Marlowe, Dido.Queene of Carthage: "poor Troy, so long suppress'd, / From forth her ashes shall advance her head, // And flourish once again that erst was dead» (1. i. 93-95) ; Heywood, dédicace de The Iron Age : "For what Pen of note, in one page or other hath not remembred Troy, and bewayl'd the sacke and subuersion of so illustrious a Citty : which, although it were scituate in $A$ sia, yet out of her ashes hath risen two the rarest $P$ hoenixes in Europe, $\mathrm{namely}$ Lond on and Rome», The Dramatic Works of Thomas Heywood, éd. R. H. Shepherd, 1874, réimp. New York, 1964, vol. 3, pp. $261-62$.

13. Quand ce n'est pas pour souligner qu'elle est comme lui unique, ou, plus rarement, pour regretter qu'elle ne puisse renaître, comme dans la médaille de 1574 (voir G. F. Hill, Medals of the Renaissance, Oxford, 1920 , p. 153; Roy Strong, Portraits of Queen Elizabeth $I$, Oxford, 1963, pp. 134-36).

14. Voir notes de A. C. Hamilton dans son éd. de The Faery Queene, Londres, 1977.

15. Distinction établie par Thomas $M$. Greene, The Light in Troy. Imitation and Discovery in Renaissance Poetry, Yale U. P., New Haven / Londres, 1982 , p. 86.

16. Ed. G. D. Willcock et A. Walker, Cambridge U. P., 1936 (1970), I. vi, pp. 11-12.

17. Ibid,, I, viii, pp, $18-20$.

18. Ibid., I. ii,, pp. $5-6$.

19. Il a traduit le dramaturge latin "from his naturall and lofty style, to our corrupt and base, or as some men (but untruly) affyrme it, most barbarous Language", Oedipus, The Epistle, in Seneca his Tenne Tragedies, éd. Thomas Newton, Londres, 1581, p. 76; que l'anglais ait besoin d'une renaissance est à nouveau suggéré dans la préface: «I bessech all together... to beare with my rudeness, \& consider the grosenes of our owne Countrey language, which can by no meanes aspire to the high lofty Latinists stile. Myne onely entent was to exhorte men to embrace Vertue and shun Vyce...». ibid., p. 78 .

20. Préface de E. K. à The Shepheardes Calender, in Spenser, Poetical Works, éd. J. C. Smith et E. de Selincourt, Oxford U. P., $1912(1970)$, p. 417 .

21. Le mythe de la Renaissance 1420-1520, Genève, 1969, pp. 8-9.

22. Sur les autres manifestations de ce thème traditionnel, voir R. Mark Benbow dans son éd. de The Araygnement of Paris 
in The Dramatic Works of George Peele, Yale U.P., New Haven/ Londres, 1970 , vol. 3, p. 20 . Il convient d'y ajouter le tableau de Hampton Court attribué à Hans Eworth (voir Roy Strong, op. cit. p. 79 et phanche 6).

Dans le livre II de The Faery Queene, Arthur et Guyon lisent chacun un ouvrage de la bibliothèque d'Eumnestes : le premier une chronique de la Grande Bretagne de Brut à Uther Pendragon, l'autre un livre intitulé Antiquitee of Faery, Lond, histoire des Elfes. Histoire et fiction sont donc ici maintenus distincts, séparés, pour étre réunis dans la prophécie de Merlin (voir Andrew Fichter, Poets Historical. Dynastic Epic in the Renaissance, Yale U.P. . New Haven/ L ondres, 1982, p. $183-84$ ).

23. Voir Panofsky, Renaissance and Renascences in Western Art, Uppsala, 1958 (Londres, 1970), pp. 10-11. Les ruines de l'ancienne Rome incitent Pétrarque à prédire un renouveau, annoncé dans 1'Africa, épopée de la seconde guerre Punique, où Scipion est victorieux des forces de barbarie que symbolise Hannibal: "Our posterity, perchance, // when the dark clouds are lifted, may enjoy // once more the radiance the ancients knew.// Then shall you see on Helicon's green slope // new growth arise, the sacred laurel bear // new leaves, and talents will spring up renewed...", Africa, IX. 639-44, ds. la trad. angl. de Thomas G. Bergin et Alice S. Wilson, Yale U.P., New Haven / Londres, 1977.

En Angleterre, le Moyen Age est loin d'etre totalement renié. $L$ 'exemple de Spenser, et le néo-médiévalisme élisabéthain le mon. trent à l'évidence. Puttenham considère comme temps de déclin l'invasion normande (op. cit., I. xxxi, pp. 59-60) et la «civilisation monastique» (ibid., I, vii, p, 12).

24. Arthur B. Ferguson, Clio Unbound, Perception of the Social and Cultural Past in Renaissance England, Duke U.P., Dur. ham, North Carolina, 1979. sig. $A 4^{\mathrm{T}}$

25. Chapman, The Georgicks of Hesiod, Londres, 1618,

$$
\text { 26. Ibid., sig. } \mathrm{A} 4^{\mathrm{V}} \text {. }
$$

27. Voir Eugenio Garin, Moyen Age et Renaissance, Paris, 1969 , pp. 159-60.

28. Hen. VIII, V. iv. 39-46. Toutes les citations de Shakespeare sont empruntées aux éds. de la série $\mathrm{New}$ Arden.

29. IHen, VI, I. i. $63-64,66-67,80$.

30. Ibid., I. vi. 6-7: "Thy promises are like Adonis' gardens, // That one day bloom'd, and fruitful were the next». Mais c'est la conquête d'Orléans qui est de courte durée ! Les jardins d'Adonis étaient connus pour dépérir aussitôt «sous l'effet de la chaleur méme qui en a favorisé la croissance hâtive» (Marcel Détienne, Les Jardins d'Adonis. La mythologie des aromates en Grèce, Paris, 1972, p. 191); il s'agit d'autre part de "cultures sans fruits et fondamenta. lement stériles» (ibid., p. 192). Voir aussi Platon, Phèdre, 276. 
31. IHen, VI, IV. v. $1-5$

32. I Hen. VI, II. iv. $85 ; 99 ; 110-11$;II. v. $41 ; 80 ; 2$ Hen. VI, II. ii. 56-57; III. ii. $212-13$; V.i. $206-207 ; 3$ Hen. VI, I. i. 48 ; III. iii. $124-26 ; 198 ;$ V. vii. $31-32$.

33. I Hen. VI, II, v. 11-13;55-57;61; 2 Hen VI, I. ii. 1-2; II. iii. 45 ; III. i. 89.90 ; III. ii. $174-75$; IV.x. 62 ; 3 Hen. VI, II. iii. 47 ; III. ii. $125-26 ; 156$; IV, iii. 23-24; V. vi, 52; V. vii. 21 .

34. 1 Hen. VI, IV . vii.1 5-16;2Hen.VI,I.ii.33;I. iii. 99; II, iii. 42 ; III : i. 31-33; 67; 3 Hen, VI, I. iii. 32;II. i. 70-71;131-32; V.v. 60 ; V. vii. 3-4. $11-15$

35. 3 Hen, VI, II. i. 54-55; II. ii. $163-69$; II. vi. 47-50; V. ii.

36. 3Hen. VI, III. ii. 174-81; V. iv. 67-71.

37. R. III, I. iii. 228 .

38. ibid., V. ii, 7-8.

39 The Faery Queene, III, iii. 42. Voir aussi A. Fichter, op. cit., pp. $177-78$.

40. Juges, 14. 8; voir A. R. Humphreys, note ds. son éd. de la pièce. Sur le symbolisme de l'abeille, voir W. De onna, "L'abeille et le roi", Revue Belge d'Archéologie et d'Histoire de l'Art, XXV (1956), pp. 105-131.

41. XXVI, xiii, p. 327 d (nous utilisons la trad, franç. de Montlyard, Ly on, Frellon, 1615 ).

42. Aussi, 1. i, $60-62$ : "The strawberry grows underneath the nettle, // And wholesome berries thrive and ripen best // Neighbour'd by fruit of baser quality».

43. Trad. H. Berthaut, Paris, 1938 .

44. Met., XV. 364-66, trad. Lafaye, Paris, 1928. Sur les origines de cette croyance, voir Déonna, loc. cit., p. 119 , note 3.

45. Voir Andrew Gurr, "Henry $V$ and the Bees' Commonwealth», Shakespeare Survey, 30 (1977), pp. 61-72; l'emb. d'Alciat, «Principis clementia», Emblemata, Anvers, Plantin, 1574, E. 148, p. 388 , et celui de Whitney, «Patria cuique chara», $A$ Choice of Emblemes, Leyden, Plantin, 1586, p. 200; voir aussi Déonna, loc. cit. , p. 117 sqq. Au Moyen Age, la ruche était emblème de la vie monastique (H. Friedmann, $A$ Bestiary for Saint Jerome. Animal Symbolism in European Religious Art, Smithsonian Institution Press, Washington, 1980, p. 152.

Dans 1 Hen. IV, Hotspur est «wasp-stung» (I. iii. 233); dans $2 \mathrm{Hen}$. $I V$, les rebelles «Have here up-swarm'd them» (IV. ii. 30). Cette image de la rébellion était déjà dans $2 H e n, V I$, III. ii. 124-26. Caractéristiquement, Henry IV a une conception faussée du rôle de son fils 
dans la communauté de la ruche (2 Hen. IV, IV. v. 74-79).

46. Ex bello pax, E. 177, op. cit., pp. $445-49$.

47. Op. cit., p, 138 .

48. Euphues and his England in The Complete Works of John Lyly, éd. R.W. Bond, Oxford U.P., 1902 (1967), vol. 2, p. 209; image reprise dans un sonnet présenté à la reine le 17 novembre 1590 (ibid., vol. 1, pp. 411-12); et dans Campaspe, IV. iii. 8 (vol. 2, p. 347), mais pour déplorer ici l'engourdissement des temps de paix.

49. John Nichols, The Progresses. . of Queen Elizabeth, Londres, 1823 , vol. 3, p. 405 .

50. Voir Déonna, loc. cit., p. 120.

51. Op. cit., V. xix, pp. 544-47.

52. Op. cit., XXVI. $x x, p .329 \mathrm{~d}$.

53. Cinq Livres des Hieroglyphiques, Paris, de He uqueville, 1614, p. 183 .

54. Panofsky, Studies in Iconology, pp. 59-67.

55. Ovide, Métamorphoses, I. 111-12.

56. Loc. cit., vo1. 2, p. 44.

57. Voir Thomas M. Greene, op. cit., pp. 68, 73,84,98-99. On mesure l'écart avec le Moyen Age lorsqu'on se souvient que dans 1'Oviđe. Moralisé, ce sont les essaims des apôtres et des précheurs qui butinent la Sainte Ecriture pour en extraire le sens de la Sainte Doctrine (XV. 5925-42, éd. C. de Boer, Amsterdam, 5 vols., 1915 . 38, vol. 5, p. 338), Le taureau d'où naissent les abeilles est le Christ mort et ressuscité (ibid).

On peut voir dans l'admiration de Fluellen pour «the Roman disciplines», transposée dans le domaine de l'art militaire, une caricature de l'imitation non créatrice. Sur les controverses élisabéthaines au sujet des traités d'art militaire, voir Lily B. Camphell, Shakespeare's Histories Mirrors of Elizabethan Policy, 1947 (Londres, 1980 ), pp. $296-305$.

58. Valeriano, op. cit., XXVI. iv, vii, xvii, pp. $324.25,329$.

59. Voir aussi Bernard Moro et Michèle Willems, "Death and Rebirth in Macbeth and The Winter's Tale, Cahiers élisabéthains, 21 (1982), pp. 35.48 .

Sans doute faudrait-il consacrer une étude aux rapports qui se nouent entre les mythes de renaissance et les thèmes de sexualité / stérilité. Jean-Marie Maguin les a étudiés dans quelques poèmes de Donne: "Le Phénix caché. Sens et statut de l'image occultée dans deux poèmes de Donne», in John Donne, éd. J.-M. Benoist, "Les Dossiers H», Paris, 1983, pp. 135-39. Dans Hamlet, comme souvent 
ailleurs (en particulier Dido Queen of Carthage) les images de miel sont liées à l'amour ( $\mathrm{Ham}$., III. i. 158, III. iv. 93). Le viol de Lucrèce a pour toile de fond la prise de Troie...

60. In John Donne, The Complete English Poems, éd. A.J. $\mathrm{Smith}, \mathrm{H}$ armondsworth, 1971 , pp. $270-83$, vers $385-86$.

61. Sur le thème du démembrement, voir aussi Ann Thompson, "Philomel in Titus Andronicus and Cymbeline», Shakespeare Survey, 31 (1978), pp. 23-32.

62. Nashe écrivait à propos des pièces historiques «our forefathers' valiant acts, that have lain long buried in rusty brass and wormeaten books, are revived, and they themselves raised from the grave of oblivion» Pierce Penniless, in The Unfortunate Traveller and Other Works, éd. J. B. Steane, Harm ondsworth, 1972 (1978), p. 113. Dans ce sens Propero est une image du dramaturge : «graves at my command // Have wak'd their sleepers, op'd, and let'em forth // By my so potent Art. 》(Temp., V.i.48-50).

63. De ce strict point de vue, Henry VIII serait peut-etre considéré comme une survivance attardée, comme le sont du point de vue formel The Misfortunes of Arthur en 1588 ou Locrine (c. 1591). A noter cependant les rapprochements avec les «Romances» établis par R. A. Foakes ds. la préface de son éd. La métaphore végétale est pessimiste dans le discours de Wolsey (III. $i$ i. 350-72), $\mathrm{m}$ ais l'im age d'Orphée optimiste dans le chant de Catherine (III. 1. 3-14). Peuton $y$ voir une opposition d'ordre éthique et religieux entre ces personnages?

64. Voir sur ce point David Young, The Heart's Forest. A Study of Shakespeare's Pastoral Plays, Yale U.P., New Haven / Londres, 1972.

65. An Elegie upon the Death of the Deane of Paul's, Dr. John Donne (1633).

66. L espace littéraire, Paris, 1955 (1982), pp. 22 7-34.

67. La civilisation de la Renaissance, Paris, 1967, p. 137.

68. Sur Orphée, voir Kirsty Cochrane, "Orpheus Applied: Some Instances of his Importance in the Humanist View of Ianguage», The Review of English Studies, n. s. 19 (1968), pp. 1-13; M. Owen Lee : "Orpheus and Eurydice: Myth, Legend, Folklore», Classica et Mediaevalia, 26 (1965), pp. $402-412$; Kenneth R.R. Gros Louis, "The Triumph and Death of Orpheus in the English Renaissance», Studies in English Literature, 9 (1969), pp.63-80; John Warden, éd., Orpheus. The Metamorphoses of a Myth, Univ. of Toronto Pr., Toronto / Buffalo / Londres, 1982. Sur la tradition médiévale : John Block Friedman, Orpheus in the Middle Ages, Harvard U.P. , Cambridge, Mass., 1970.

69. Boccace, Genealogie Deorum Gentilium Libri, XIV. viii, éd. V. Romano, Bari, 1951 , vol. 2, pp. $701-05$; Conti, op. cit., VII. xiv, pp. 765-72; Golding, The $X V$ Bookes of $P$. Ouidius Naso, 
entytuled Metamorphosis, Epistle, 517-26 (éd. Rouse, Londres, 1961, p. 11). Wills, De Re Poetica (1573) éd. et trad. A.D.S. Fowler, Oxford, 1958, pp. 61-62; Lodge, Defence of Poetry (1579), in G. Smith, éd., Elizabethan Critical Essays, Oxford U.P., 1904, p. 73 sqq.; We bbe, A Discourse of English Poetrie (1586), éd. Arber, Lon. dres, 1870 , p. 25; Puttenham, op. cit., I. iii, p. 6; Sidney, op. cit., pp. 18-19. La source est Horace, Art Poétique, 391-99.

70. The Shepheardes Calender, Oct., 28; The Ruines of Ti. me, 390-92; The Faery Queene, IV.x. 58. p. 153.

71. Eugenio Garin, Moyen Age et Renaissance, Paris, 1969,

72. Voir P.O. Kristeller, Renaissance Thought and its Sources, Columbia U.P., New Y ork, 1979, chap. 10, pp. 196-210.

73. Loc. cit., $112-204$

74. Ibid.,191-92;Ham.,I. v, 196.

75. Ibid., 213

76. Loc cit.

77. Ibid., 215-18.

78. Ibid., 473-74

79. Nous suivons l'analyse de Gros Louis (loc. cit.), qui cite Drayton, Davies, Marston, etc...,p. 73 sqq.

80. The Wisedome of the Ancients, Londres, 1619, p. 60 . 